method up to now. In all of them freedom from staphylococci was achieved, and they improved clinically.

\section{Conclusion}

The first part of these studies shows, that the inhalation of methicillin particles leads to the total eradication of staphylococci as a source of cross-infection. This result cannot be achieved by any of the methods previously described. In spite of predictions to the contrary, the emergence of drug resistance and hypersensitivity phenomena have not been found.
In the second part of these studies, that is the treatment of staphylococcal bronchitis by the inhalation of methicillin, a successful result was achieved.

Finally we have made some financial comparisons, and these have shown that the various methods of spraying are considerably cheaper, than is the cost of maintaining infected patients in hospital.

\section{REFERENCES}

Elek, S. D., and Fleming, P. C. (1960a), Lancet, i, 1009.

Elek, S. D., and Fleming, P. C. (1960b), Ibid., ii, 569 .

\title{
CLINICAL EXPERIENCES WITH THE PROPHYLACTIC AND THERAPEUTIC USES OF THE NEW PENICILLINS IN CARDIOVASCULAR-RENAL DISEASES
}

\author{
ELLARD M. Yow
}

Infectious Disease Section, Department of Internal Medicine, Baylor University College of Medicine, and the Infectious Disease Laboratory, Methodist Hospital, Houston, Texas.

THE treatment of a staphylococcal infection complicating the implantation of cardiovascular prostheses has almost always failed unless the prostheses were removed. Even if the prostheses were removed and a new one implanted in an infected field, the procedure was usually unsuccessful. The greatest success has been in those cases in which it was possible to remove the prosthesis, adequately treat the infection, and re-implant the graft in a sterile field. However, removal of a graft in the aorta or a prosthetic heart valve is not always compatible with life for sufficient periods of time to allow adequate treatment of the infection.

While infection of cardiovascular prostheses is not common (though it was reported to be as high as $10 \%$ at one clinic) (Geraci, Dale and McGoon, 1963), when it does occur it is usually life threatening. The difficulties encountered in the management of these infections when they do occur made us consider more seriously chemoprophylaxis as a possible approach to this problem. Consequently, three years ago a programme was instituted in which all patients having cardiovascular surgery in two of the four hospitals in the Texas Medical Center doing this type of surgery were given methicillin 6 grams daily and streptomycin
1 gram daily for two days before and four days following surgery. Those patients giving a history of allergy to penicillin were excluded from the study.

A rather complex statistical analysis of the results of this study is now being carried out, but since several thousand cardiovascular operations are involved it will require some time for completion. It is of interest, however, that the information already available reveals that there have been no proved cases of serious staphylococcal infections in the methicillin treated group, whereas there have been several deaths among the control groups in whom infections played a major role.

The use of methicillin prophylactically has not prevented all infections. There have been several patients who developed Gram-negative bacillary infections, notably pseudomonas, but none of these have been as difficult to handle as have the staphylococcal infections.

The same prophylactic régime was used in four patients receiving kidney homotransplantations and two additional patients known to be allergic to penicillin received cephalothin rather than methicillin. There have been no infections in this group of patients.

While it seems likely at this stage that the 
prophylactic use of methicillin has decreased the incidence of staphylococcal infections in the cardiovascular surgical patients, there has been some concern about the possibility of selectively increasing the resistance of hospital strains of staphylococci to this antibiotic. Consequently, one year ago a programme was instituted in which all strains of staphylococci isolated from patients with infections in the hospital (Methodist) in which the prophylactic régime was most frequently used were tested quantitatively for susceptibility to penicillin $G$, methicillin, oxacillin and cephalothin. The results of these tests are graphically presented in Figures 1, 2, 3 and 4, and do not indicate any increased resistance to either of the penicillinase-resistant penicillins.

Finally, I would like to present several examples of the successful management of severe staphylococcal infection in patients having cardiovascular surgery.

\section{Case 1 (L.A.B.)}

On February 4th, 1958, a 46-year-old man with diabetic nephropathy had a dacron aorto-femoral bypass performed for Leriche syndrome. Approximately six weeks later he began to complain of abdominal pain, chills and fever. He was re-explored and a large infected hematoma was found in the retroperitoneal area, surrounding the graft. A penicillinase producing staphylococcus was cultured from the hematoma. The wound was debrided, the old graft was removed and a new graft put in place. The area was saturated with th neomycin-bacitracin solution. Subsequently the patient was given the following antibiotics:

$\begin{array}{lll}\text { Kanamycin } & 2 \text { grams daily } & 3 / 20-3 / 25 \\ \text { Vancomycin } & 4 \text { grams daily } & 3 / 25-4 \text { days } \\ \text { Kanamycin } & 2 \text { grams daily } & 4 / 9-4 / 19 \text { days } \\ \text { Kanamycin } 1 \text { gram daily } & 4 / 19-4 / 25-6 \text { days }\end{array}$

During therapy the patient's blood urea was as high as $70 \mathrm{mg}$. per cent. He had no evidence of relapse of the infection and his graft has continued to function well for the six years since his last surgery though he has almost total loss of hearing.

\section{Case 2 (G.B.)}

A 47-year-old man had an aorto-bilateral iliac by-pass procedure carried out in another city in July, 1960. In February of 1961, because of evidence of further occlusive disease, the grafts were extended to the popliteal arteries. This was followed by rupture along a suture line and re-exploration revealed evidence of infection involving the aortic end of the graft. The graft was removed and another put in place. In spite of antibiotic therapy the wound drained purulent material and a culture of the exudate revealed a penicillinase-producing staphylococcus.

In July, 1961, he was transferred to the Methodist Hospital in Houston. A fourth operation was carried out here, and the infection was found to extend into the retroperitoneal space alongside the graft. The wound was debrided but the graft was left in place, polyethylene catheters were implanted in the solution was dripped into the catheters over $\mathbb{Q}_{a}$ period of one month.

He received the following antibiotic therapy:

$$
\begin{aligned}
& \text { Methicillin : } \\
& 12 \text { grams daily }-10 \text { days } \\
& 8 \text { grams daily }-7 \text { days } \\
& 4 \text { grams daily }-2 \text { months } \\
& \text { Diphenicillin : } \\
& 4 \text { grams daily }-1 \text { month } \\
& 2 \text { grams daily }-2 \text { months }
\end{aligned}
$$

The wound ceased to drain and had healed the beginning of the second month of therapy.

In February of 1964, the patient returned Houston for a carotid endarterectomy. At this tiofe the aorto-popliteal by-pass was functioning well a d there was no evidence of infection.

Case 3 (J.A.)

A 45-year-old man developed a staphylococca endocarditis following surgery for the correction stenosis of the aortic valve. He was treated postoperatively with chloramphenicol. However, withincta few days of the chloramphenicol being discontinued he became febrile and a coagulase-negative staphyFcoccus was isolated from his blood stream. The

\begin{tabular}{|c|c|c|}
\hline Antibiotic & $\begin{array}{c}\text { Minimal } \\
\text { Inhibitory } \\
\text { Concentration } \\
\mu \mathrm{g} . / \mathrm{ml} \text {. }\end{array}$ & $\begin{array}{r}\text { Minimg } \\
\text { Bactericg } \\
\text { Concentrat } \\
\mu \mathrm{g} . / \mathrm{ml}\end{array}$ \\
\hline $\begin{array}{l}\text { Penicillin G } \\
\text { Phenethicillin } \\
\text { Methicillin } \\
\text { Kanamycin } \\
\text { Vancomycin } \\
\text { Streptomycin }\end{array}$ & $\begin{array}{l}0.10 \\
0.78 \\
6.25 \\
0.20 \\
1.56 \\
20.0\end{array}$ & $\begin{array}{l}0.20 \\
0.78 \\
6.25 \\
0.78 \\
3.12 \\
20.0\end{array}$ \\
\hline
\end{tabular}
strain was found to be sensitive in vitro to most antibiotics including chloramphenicol. Results of the sensitivity tests using the bactericidal antibiotics a shown in Table I.

Table I. (J.A.) Antibiotic Sensitivity Tests-

Because of the seriousness of the disease and $i$ recent history of a severe allergic reaction to penicislin, he was treated with both kanamycin and vancemycin. His blood cultures promptly became sterie, but within a few days after the antibiotics were discontinued and while he was receiving erythromycin, his fever and bacteremia returned. The stram of staphylococcus isolated at this time was identical in all respects to the original strain. He was subsequently treated with methicillin and streptomycip Cortisone was also given because of the evidence history of allergy to penicillin. There was a prom $\bar{p}$ clinical and bacteriologic response, and after thir days the treatment was discontinued.

Three weeks later there was a relapse with a retu㑤 of the fever, and a staphylococcus with the sanng characteristics was isolated from the patient's bloof The dose of methicillin was then increased to 2 grams daily with 20 grams administered by slow intravenous drip and 2 grams rapidly given intravenously. The patient's serum showed a bactericids effect against his organism in a dilution of $1: 4$. This regimen was continued for four weeks and followes 


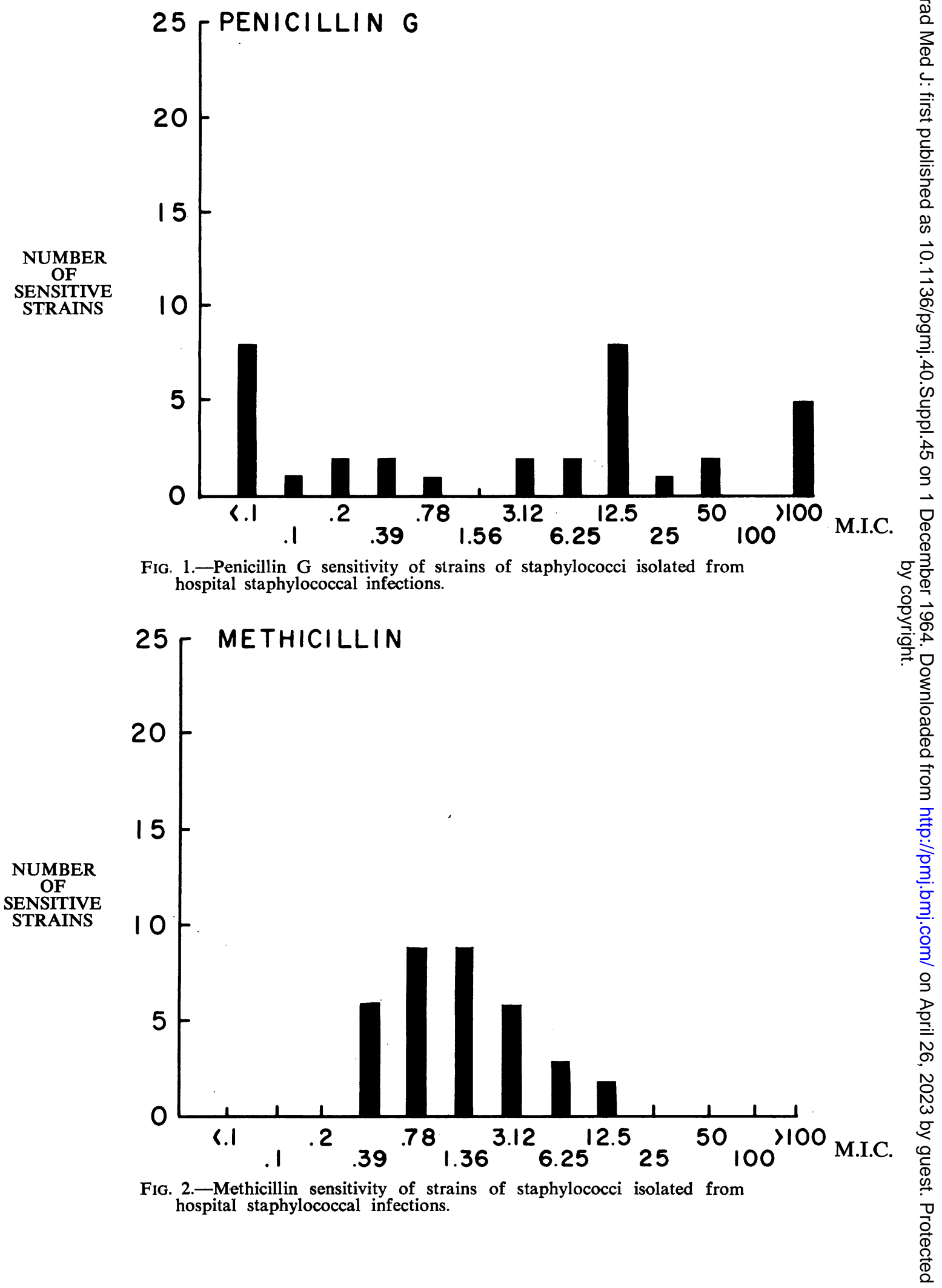




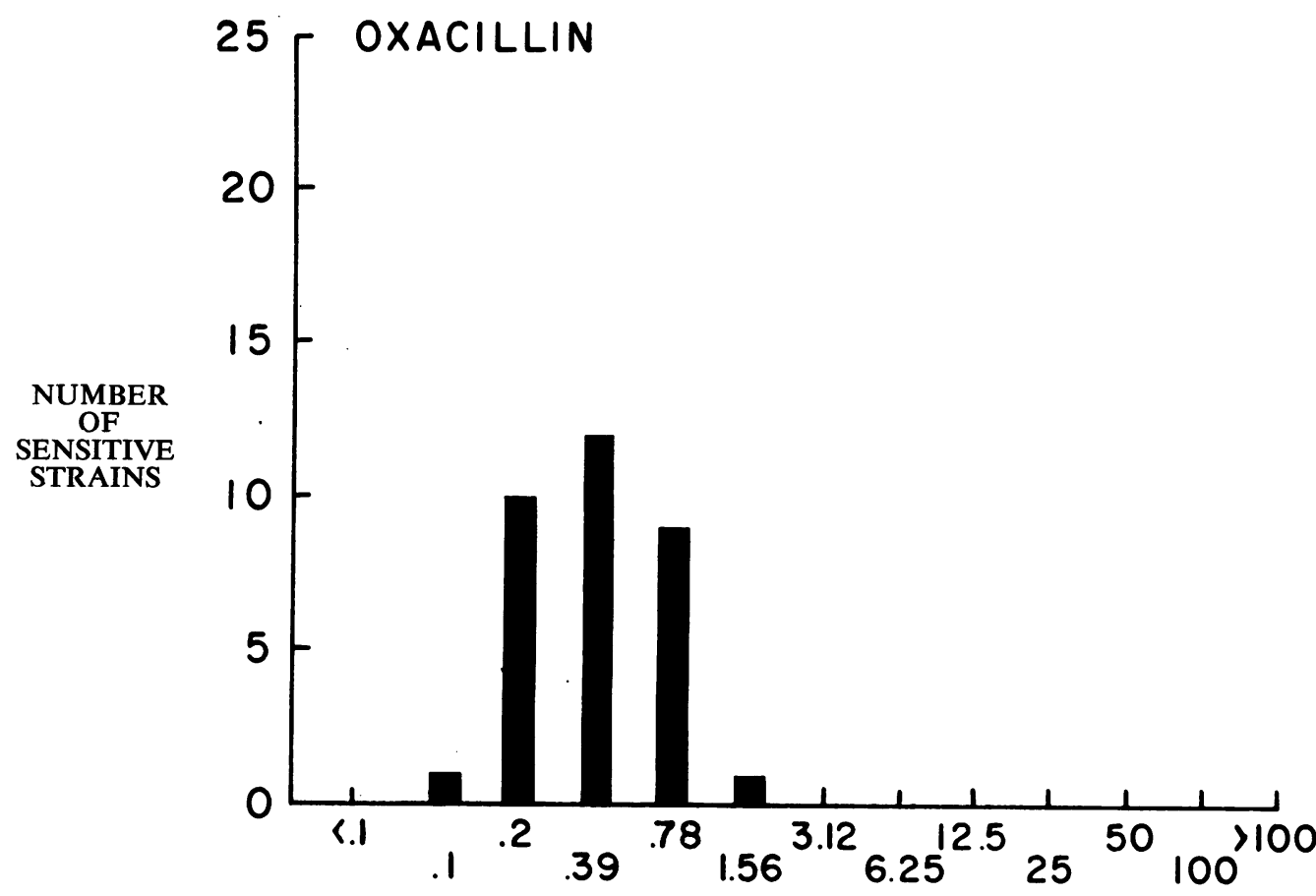

FIG. 3.-Oxacillin sensitivity of strains of staphylococci isolated from hospital staphylococcal infections.

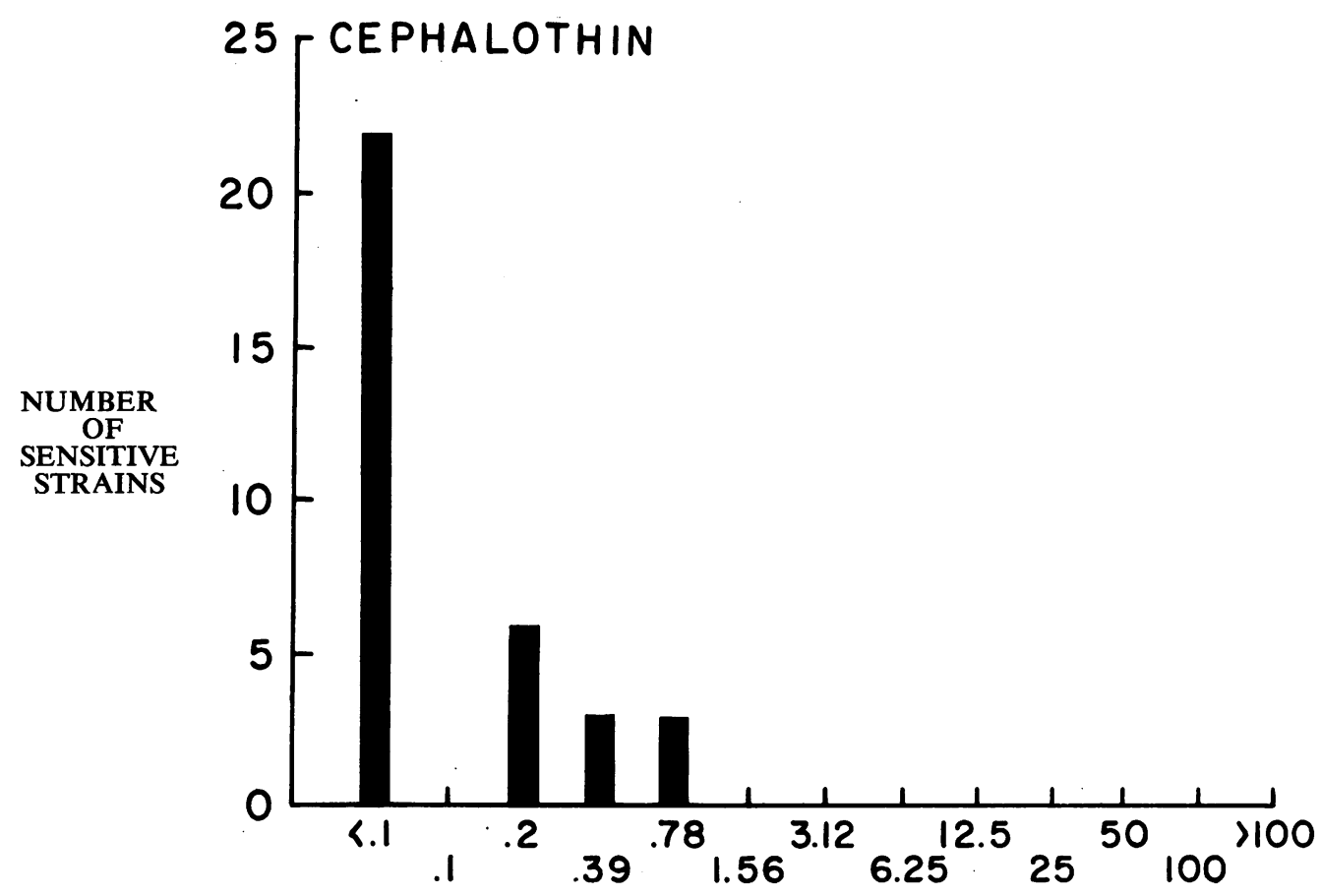

FIg. 4.-Cephalothin sensitivity of strains of staphylococci isolated from hospital staphylococcal infections. 
by the intramuscular administration of 2.0 grams daily for four weeks. Cessation of therapy was followed by another relapse without evidence of increasing antibiotic resistance of the causative organism. He was subsequently treated for 106 days with doses varying from 0.5 grams to 20 grams daily. Although the smaller dose controlled the fever and bacteremia for 42 days, relapse ultimately recurred during therapy. The patient was then placed on 45 grams daily by intravenous drip. There were no complications of therapy in this dosage range except for the development of a thrombophlebitis at the site of the infusions and excessive fiuid retention as the result of the large amount of sodium administered in the methicillin salt. Each infusion was given over an eight hour period to prevent the deterioration of the methicillin to the therapeutically inactive penicilloic acid. Measurement of the serum cidal power during this course of methicillin revealed that the serum killed the causative organism in dilutions of from $1: 16$ to $1: 64$. After four weeks of therapy, the drug was discontinued. The patient has been followed for three years and there has been no evidence of recurrence of infection.

\section{Case 4 (D.H.)}

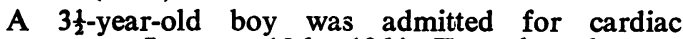
surgery on January 13 th, 1964. Two days later a ventricular septal defect was repaired with a dacron patch. Post-operatively he had a high fever in spite of penicillin and streptomycin therapy (Figure 5). The fever continued and blood cultures were positive for Staphylococcus aureus on January 24th, 1964. Subsequently blood cultures drawn on January 27 th and 28th were also positive. On January 28th the patient appeared very ill, he had pain in the right knee and could not straighten the leg because of pain on extension. There was an intermittent cardiac friction rub and there was hepatomegaly. The spleen was larger and tender.

Anti-staphylococcal treatment was initiated using methicillin on January 28th, 1964 . Blood cultures drawn on January 30 th were sterile and all subsequent cultures remained sterile. The patient began to feel subjectively improved within 48 hours. On January 31st a fluctuant area appeared in his suture line. This area yielded approximately $10 \mathrm{cc}$. of purulent material which was positive for Staphylococcus aureus.

Antibiotic sensitivity tests on this organism and the organism obtained from the blood stream were identical. The results of the tube dilution tests were as follows :

Antibiotic
Penicillin G
Methicillin
Oxacillin
Cloxacillin
Minimal Bactericidal Concentration $25 \mathrm{mcg} / \mathrm{ml}$. $6.25 \mathrm{mcg} / \mathrm{ml}$. $1.56 \mathrm{mcg} / \mathrm{ml}$. $1.56 \mathrm{mcg} / \mathrm{ml}$.

The patient continued to have fever in spite of this drainage and high doses of methicillin but he felt better and the leg pain disappeared. On February 4th an aneurysm of the right femoral artery was detected. This was removed and an end-to-end anastomosis accomplished. Following this surgery

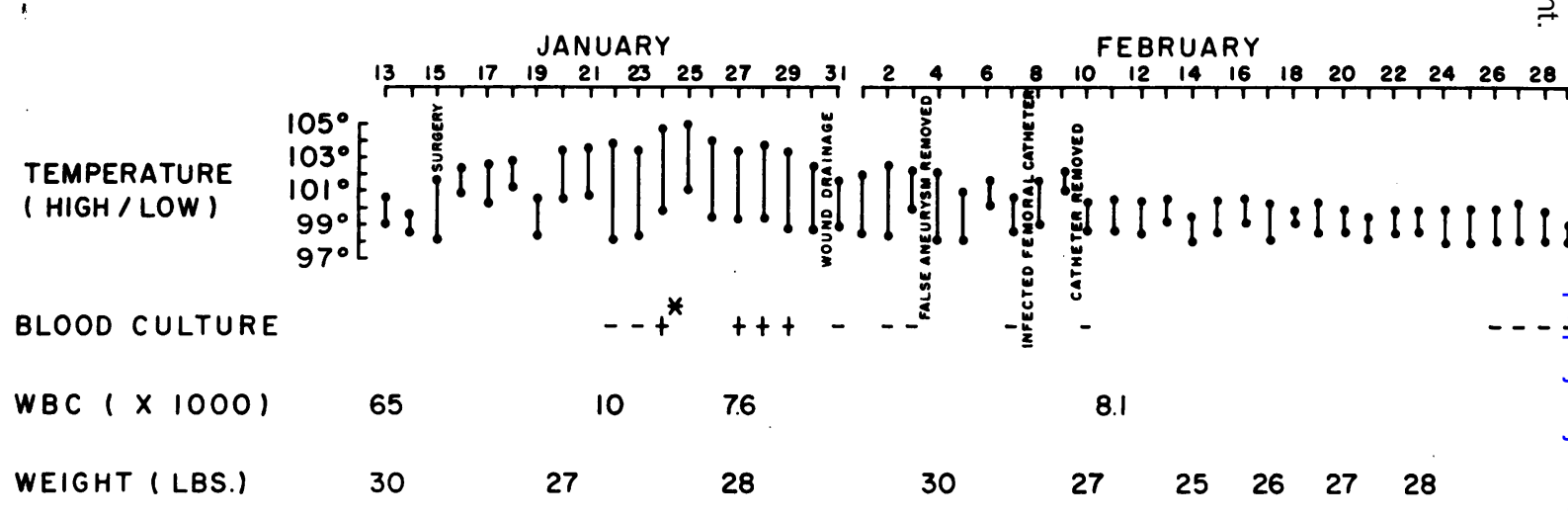

PENICILLIN G(U X10\% d $\quad 222222$

STREPTOMYCIN (GMS./d) $\quad \mathbf{5} .5 .5 .5 .5 .5$

METHICILLIN (GMS. / d)

41010101010888888844442222222222

SERUM CIDAL TITER

$\begin{array}{llll}1: 2 & 1: 1 & 1: 8 & 1: 64\end{array}$

* STAPHYLOCOCCUS AUREUS

Fig. 5.-Hospital course of a $3 \frac{1}{2}$-year-old boy following correction of a ventricular septal defect with dacron patch. 
the patient's fever was definitely less except for a two-day spike which was caused by an infection of a polyethylene catheter in the left femoral (this catheter was being used for administration of methicillin). The catheter was removed and cultured. It was infected by a strain of Aerobacter aerogenes. Removal of the catheter reduced the fever. Blood cultures never became positive for Aerobacter aerogenes. After the catheter was removed the patient became afebrile and remained so until his discharge from the hospital.

Methicillin was continued for two weeks after his temperature returned to normal. During this time the splenomegaly receded, the patient's appetite improved, he gained weight, the WBC became normal, and he became very active. On February 24th methicillin was discontinued. Three blood cultures were obtained on February 26th and one daily thereafter. These cultures were all negative. The patient has remained well for the four months since discharge and attends school regularly.

\section{Case 5 (J.W.M.) (Figure 6)}

43-year-old man with rheumatic heart disease was admitted to Ben Taub General Hospital on August 20th, 1963, for the treatment of aortic stenosis and insufficiency. The patient's deformed valve was replaced by a Starr valve and his postoperative course was uneventful. One month later he was admitted to the hospital because of gastric hemorrhage. He was transfused and discharged when the bleeding ceased. Blood cultures taken on this 3 admission were negative and the patient was afebrile. $\stackrel{\mathbb{Q}}{\alpha}$

One month later the patient was re-admitted to the hospital because of chills and fever. Six blood cultures were obtained and they were all positive for Staphylococcus epidermidis. The antibiotic sensitivity tests were as follows :

$\begin{array}{lcc}\text { Antibiotic } & \text { M.I.C. } & \text { M.B.C. } \\ \text { Penicillin G } & 0.10 & 0.10 \\ \text { Cephalothin } & 0.39 & 0.39 \\ \text { Methicillin } & 6.25 & 6.25 \\ \text { Oxacillin } & 0.39 & 0.39 \\ \text { Cloxacillin } & 0.78 & 0.78 \\ \text { Ampicillin } & 0.20 & 0.20 \\ \text { Nafcillin } & 0.39 & 0.78\end{array}$

On October 18th, 1963, the patient was begun on $\overrightarrow{0}$ an intravenous drip of penicillin, receiving 50,000,000 units daily for 46 days. His blood cultures became negative immediately and his serum cidal titer waso as high as $1: 256$.

On January 4th, 1964, the patient had a recurrence 을 of gastric bleeding and a gastric re-section was per- formed. Blood cultures on this admission were negative and he had no fever. On March 5th, 1964, 1

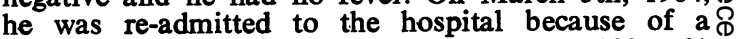
hemolytic anemia (bilirubin $4.8 \mathrm{mg}$. per $100 \mathrm{mb}$ ) thought to be due to a leak around the base of the Starr valve which had been present since the dify of operation.

ENDOCARDITIS

COMPLICATING STARR VALVE

OCTOBER

TEMPERATURE

(HIGH/LOW)

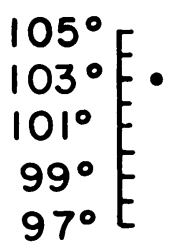

\section{8}

$\begin{array}{llllllllllll}18 & 19 & 20 & 21 & 22 & 23 & 24 & 25 & 26 & 27 & 28 & 29\end{array}$

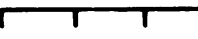


On April 13th, 1964, the aorta was re-opened, the valve inspected and aortic ring around the base of the valve tightened. The valve appeared normal at the time of operation. Following the operation the diastolic murmur disappeared and hemolysis ceased. The patient has since remained well.

\section{Case 6 (A.R.)}

A 28-year-old woman was admitted to Jefferson Davis Hospital on July 18th, 1962, for mitral anuloplasty because of progressive cardiac failure. Her past history revealed that in June of 1961 she was in the same hospital for a period of two months because of an acute staphylococcal endocarditis secondary to septic abortion. Initial treatment consisted of chloramphenicol, penicillin G, kanamycin and corticosteroids. These were ineffective in sterilizing the blood stream. Subsequently she was given a 30-day course of methicillin and oxacillin which effected a bacteriologic cure. Cardiac catheterization revealed findings consistent with right and left ventricular failure, mitral regurgitation, and severe pulmonary hypertension.

The patient was re-admitted July 18th, 1962, because of progressive cardiac decompensation. On July 23rd, a mitral annuloplasty was performed and immediately post-operatively the patient was placed on penicillin $\mathbf{G}$ and streptomycin prophylactically (Figure 7). The patient developed fever on the seventh post-operative day which was considered to be due to the post-pericardiotomy syndrome. Antibiotics were discontinued and salicylate therapy started. The patient improved and on the 18 th post-operative day the salicylates were discontinued. Four days later, however, the patient became febrile again. Blood cultures showed no bacterial growth. Over the next few days cardiac tamponade developed and a pericardiocentesis was done and a thousand $\mathrm{ml}$. of purulent fluid was removed. Cultures of this fluid revealed a coagulase positive Staphylococcus aureus which was resistant to $50 \mu \mathrm{g} . / \mathrm{ml}$. of penicillin $\mathrm{G}$ but susceptible to the penicillinase-resistant penicillins. Methicillin therapy was started in doses of 14 grams per day and she was given oral probenicid in doses of 2 grams a day. The serum bactericidal level on this dosage was $1: 32$. A second pericardiocentesis was performed three days after the first and a staphylococcus was again isolated. Methicillin in a dose of $50 \mathrm{mg}$. was instilled intrapericardially without undesirable side effects. A third pericardiocentesis on the 37th post-operative day was sterile. The bactericidal titer of the pericardial fluid was $1: 16$ In spite of the antibiotic therapy and repeated pericardiocenteses the patient's condition did not significantly improve and she continued to be febrile. On the 39th post-operative day a pericardiotomy was carried out for the purpose of surgical drainage. Two hundred $\mathrm{ml}$. of purulent fluid were removed from loculi over the right atrium and adjacent right ventricle. The remainder of the heart was covered by a pericardial rind one $\mathrm{cm}$. thick. Partial pericardiectomy was performed. Following the pericardiectomy the course was uneventful and intravenous methicillin therapy was discontinued on the 57th hospital day and oxacillin was given orally in a dose of two grams daily. The patient has subsequently remained well.

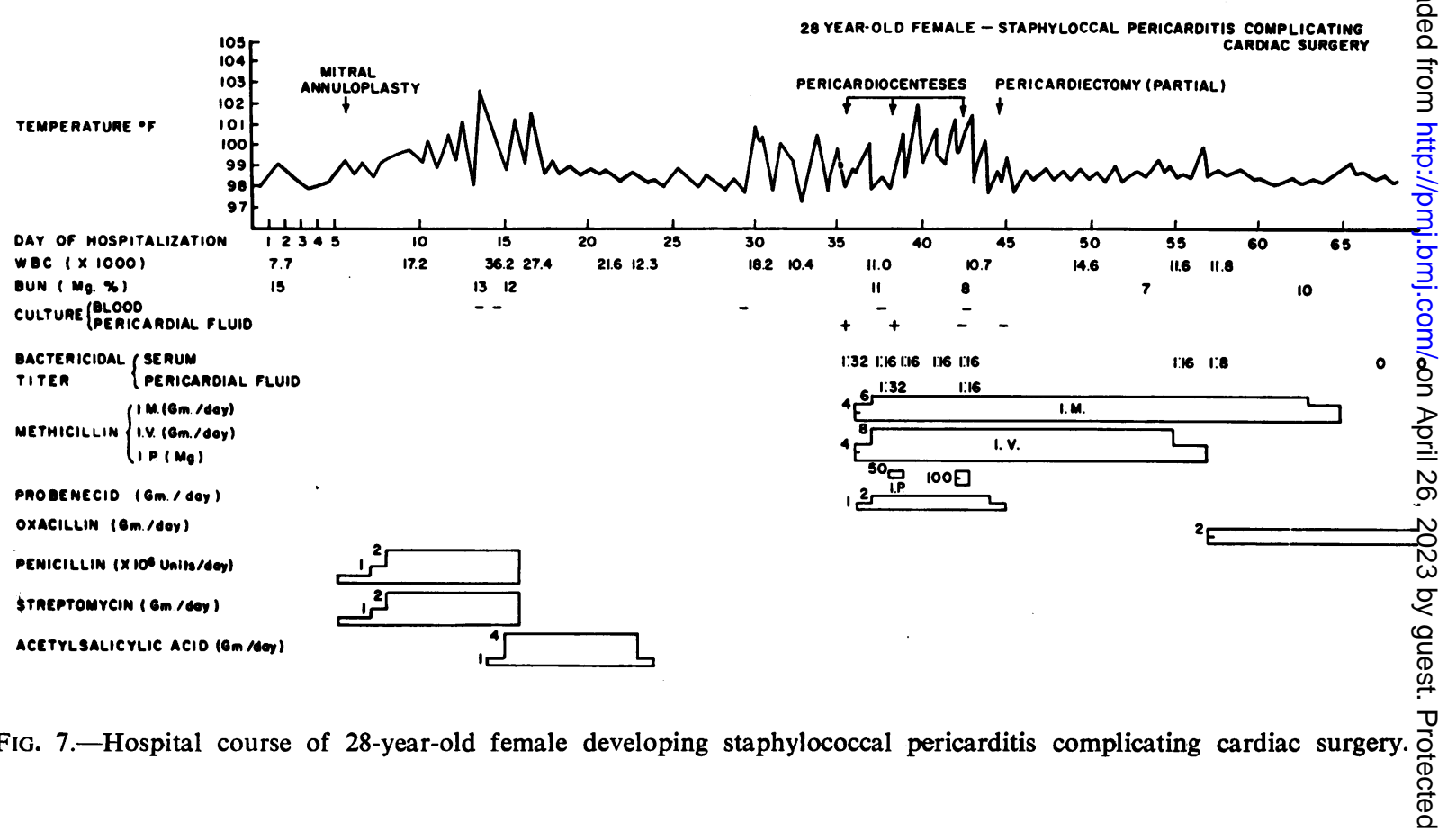




\section{Summary}

Staphylococcal infections complicating cardiovascular prostheses are associated with a grave prognosis. Consequently, the prophylactic use of methicillin in this group of operations seems justified. Occasionally such infections can be successfully managed without removing the prosthesis by intensive and prolonged local and systemic therapy with appropriate bac- $\stackrel{\mathbb{Q}}{\stackrel{\mathrm{Q}}{2}}$ tericidal antibiotics.

\section{REFERENCE}

Geraci, J. E., Dale, A. J., and McGoon, (1963): Wis. med. J., 62, 302.

\section{DISCUSSION}

Chairman: B. C. Stratford (Melbourne); Participants: W. M. M. KIRBY (Seattle);

M. Barber (London); C. A. Green (Newcastle-upon-Tyne); L. D. Thrupp

(Los Angeles); P. Naumann (Hamburg).

\section{Alternative Therapy in Patients Hypersensitive to Penicillin.}

KIRBY: I would say that many patients who are thought to be sensitive to penicillin are not sensitive, and I think that depending on how pressing a situation it was I would first try a small dose of penicillin $G$, having adrenalin handy, because often if one starts out with one unit intramuscularly or intravenously and increases the dose to 20 million units, working up within a few hours, there is no reaction. I think these are merely people in whom the sensitivity reaction does not occur as it allegedly did before. We have done this many times.

If I were convinced, by getting reactions, that penicillin could not be used, then the second choice at the present time would be cephalothin. In our series of about 150 patients we have seen no cross-reactions with cephalothin. It has a certain series of reactions itself and if it was causing reactions I would then have, as a third choice, vancomycin.

We have had very limited experience with fucidin. In the old days we had a great deal with novobiocin, and these drugs are very similar in their extremely high serum binding and the fact that staphylococci become resistant with a single exposure. Our own experience with these drugs for reasons that they have very little free antibiotic and develop resistance early has not been favourable.

BARBER: In spite of all the laboratory tests which suggest it would be very protein bound, in using fucidin we have been quite staggered at how effective it is clinically and it is often our second choice.

GREEN: I can support that last remark. We 9 have been using fucidin repeatedly in these $D$ cases, in combination with another drug, and this is our main measure of support. Un- N fortunately we occasionally have a super infection with Gram negative organisms and our finding in these cases is that we have totally failed to cure the Gram negative infection.

THRUPP: One clinical problem leading to failure of the new penicillin treatment for pulmonary

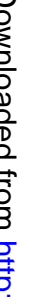

